\title{
Cobertura vacinal das gestantes em Ponte Nova - MG e fatores associados
}

\author{
Vaccine coverage of pregnant women in Ponte Nova - MG and associated factors \\ Cobertura vacuna de mujeres embarazadas en Ponte Nova - MG y factores asociados
}

Luana Silva de Andrade ${ }^{1 *}$, Ruth Maria Alves Garcia ${ }^{1}$.

\begin{abstract}
RESUMO
Objetivo: Estimar a Cobertura Vacinal (CV) dos gestantes no município de Ponte Nova - MG. Métodos: Trata-se de um estudo descritivo, quantitativo e retrospectivo realizado através da coleta de dados fornecidos pelo Departamento de Informática do Sistema Único de Saúde (DATASUS) - TABNET entre os anos de 2018 e 2020. Resultados: A CV das gestantes no município mineiro seguiu um padrão de queda em praticamente todos os anos analisados, sendo que a vacina da Influenza uma diminuição de 34,49\% entre os anos de 2020 , o mesmo ocorreu com a dT e com a dTpa gestante que teve uma queda de aproximadamente $44 \%$ quando comparados os anos de 2018 e 2020, já a dTpa gestante preconizada a todas as gestantes apresentou queda de $24,15 \%$ entre os anos de 2018 e 2020 . Por fim, a CV da hepatite B para toda a população -alvo apresentou redução de 26,82\% de 2018 para 2019 e elevação entre 2019 e 2020 de 30,51\%. Conclusão: Constatou-se que a situação vacinal gestantes no município de Ponte Nova - MG e também no território brasileiro está abaixo dos valores de cobertura preconizado pelas entidades de saúde.
\end{abstract}

Palavras-chave: Vacinação, dTpa/dT, Hepatite B, Influenza, Gestantes.

\section{ABSTRACT}

Objective: To estimate the Vaccination Coverage (CV) of pregnant women in the city of Ponte Nova - MG. Methods: This is a descriptive, quantitative and retrospective study carried out through the collection of data provided by the Information Technology Department of the Unified Health System (DATASUS) - TABNET between 2018 and 2020. Results: The VC of pregnant women in the municipality of Minas Gerais followed a pattern of decline in practically every year analyzed, with the Influenza vaccine having a decrease of $34.49 \%$ between the years of 2020 , the the same occurred with $d T$ and pregnant dTpa, which had a decrease of approximately $44 \%$ when comparing the years 2018 and 2020, while the pregnant dTpa recommended for all pregnant women showed a decrease of $24.15 \%$ between 2018 and 2020 Finally, the CV of hepatitis B for the entire target population showed a reduction of $26.82 \%$ from 2018 to 2019 and an increase between 2019 and 2020 of $30.51 \%$. Conclusion: It was found that the vaccination situation in pregnant women in the city of Ponte Nova - MG and also in the Brazilian territory is below the coverage values recommended by health entities.

Key words: Vaccination, dTpa/dT, Hepatitis B, Influenza, Pregnant women.

\section{RESUMEN}

Objetivo: Estimar la Cobertura de Vacunación (CV) de gestantes en la ciudad de Ponte Nova - MG. Métodos: Se trata de un estudio descriptivo, cuantitativo y retrospectivo realizado mediante de la recolección de datos proporcionados por el Departamento de Tecnología de la Información del Sistema Único de Salud. (DATASUS) - TABNET entre 2018 y 2020. Resultados: La CV de gestantes del municipio de Minas Gerais

\footnotetext{
${ }^{1}$ Faculdade Dinâmica do Vale do Piranga (FADIP), Ponte Nova - MG.
}

*E-mail: luanaandrade211@hotmail.com

SUBMETIDO EM: 9/2021 ～～ACEITO EM: 10/2021 ｜ PUBLICADO EM: 11/2021


siguió un patrón de descenso en prácticamente todos los años analizados, teniendo la vacuna Influenza una disminución del $34,49 \%$ entre los años de 2020 , la lo mismo ocurrió con la dT y la dTpa embarazada, que tuvo una disminución de aproximadamente 44\% al comparar los años 2018 y 2020, mientras que la dTpa embarazada recomendada para todas las embarazadas mostró una disminución del $24,15 \%$ entre 2018 y 2020 Finalmente, el CV de la hepatitis B para toda la población objetivo mostró una reducción del $26,82 \%$ de 2018 a 2019 y un aumento entre 2019 y 2020 de 30,51\%. Conclusión: Se encontró que la situación de vacunación en mujeres embarazadas en la ciudad de Ponte Nova - MG y también en el territorio brasileño está por debajo de los valores de cobertura recomendados por las entidades de salud.

Palabras clave: Vacunación, dTpa / dT, Hepatitis B, Influenza, Mujeres embarazadas.

\section{INTRODUÇÃO}

A assistência ao pré-natal compreende a aplicação de condutas clínico-obstétricas que têm como objetivo a manutenção da integridade das condições de saúde materna e fetal; devendo ser iniciada precocemente e com atendimentos regulares durante o período gestacional. Visando a assistência de qualidade, dentre os cuidados e procedimentos preconizados durante o pré-natal, podemos destacar a garantia da vacinação das gestantes (MINISTÉRIO DA SAÚDE, 2013).

A vacinação possui um papel extremamente importante durante o período gestacional, uma vez que constitui uma ação prioritária, eficaz e estratégica da Atenção Primária de Saúde (APS) ao proporcionar a proteção materna e do concepto contra infecções e possíveis complicações, tais como, a ocorrência de infecções neonatais, malformações fetais e o óbito fetal ou neonatal. A imunização materna, através passagem de anticorpos via transplacentária e por meio da amamentação, também confere ao RecémNascido (RN) e/ou lactente proteção a certos agentes infecciosos nos primeiros meses de vida quando há maior vulnerabilidade do seu sistema imunológico (LOUZEIRO EM, et al., 2014).

Quando se trata de vacinação, o programa brasileiro, criado no ano de 1973, conhecido como Programa Nacional de Imunizações (PNI) possui reconhecimento mundial e excelência no campo da imunização por of erecer gratuitamente uma quantidade extensa de imunológicos para toda população e, consequente, pelos resultados positivos alcançados. Através do planejamento, capacitação e aplicação de inúmeras políticas e estratégias, o PNI, visa de alcançar coberturas vacinais cada vez maiores e promover, dessa forma, a erradicação e controle de várias doenças imunopreveníveis através da vacinação como, por exemplo, o sarampo, o tétano, a difteria e a coqueluche (MINISTÉRIO DA SAÚDE, 2003).

Especificamente para o grupo das gestantes, o Ministério da Saúde, através do Programa Nacional de Imunizações (PNI), disponibiliza quatro tipos de imunobiológicos: a vacina anti-influenza, que é of ertada durante campanhas anuais de imunização; a anti-hepatite $B$; a dupla adulto (dT) que será indicada a depender da situação vacinal da gestante e a tríplice bacteriana acelular (dTpa) da gestante contra a difteria, o tétano e a coqueluche (MINISTÉRIO DA SAÚDE, 2020). Todos os imunógenos preconizados e administrados durante o período gestacional seguem um esquema específico comprovadamente seguro e eficaz tanto para a mãe quanto para o feto (LAJOS GJ, et al., 2017).

Perante o exposto, este trabalho teve como objetivo avaliar a cobertura vacinal das gestantes do município de Ponte Nova/MG durante os anos de 2018, 2019 e 2020, em comparação com os dados nacionais do mesmo período. Os índices quantitativos de imunização coletados e apresentados nesta obra servirão para ampliar as discussões futuras sobre o assunto e suplementar os estudos científicos previamente publicados voltados a esta temática. Além disto, este estudo também buscou apresentar e discutir alguns dos fatores que influenciam diretamente a adesão das gestantes à vacinação.

\section{MÉTODOS}

Trata-se de um estudo descritivo, quantitativo e retrospectivo com foco na avaliação dos índices de imunização materna na cidade de Ponte Nova/MG. Para isso, foi realizada uma busca referente à cobertura 
vacinal das gestantes no município durante os anos de 2018, 2019 e 2020 segundo os registros do Programa Nacional de Imunização (PNI) disponibilizados através do Departamento de Informática do Sistema Único de Saúde (DATASUS) - TABNET.

Após a coleta e tabulação dos dados, foi estruturada uma análise descritiva sobre os índices de cobertura vacinal das gestantes identificadas no município de Ponte Nova/MG, frente às metas estabelecidas para cada uma das vacinas preconizadas pelo Ministério da Saúde e disponibilizadas durante o período gestacional, sendo elas: a hepatite $B$, influenza, dT e dTpa. Foi também realizada uma análise comparativa entre os índices verificados no município e os índices nacionais de cobertura vacinal das gestantes.

Para construção e embasamento teórico do trabalho foi realizada uma busca bibliográfica sobre a imunização e a cobertura vacinal das gestantes. Para tal, foram feitas consultas virtuais às bases de dados Scientific Electronic Library Online (SciELO), Literatura Latino-Americana e do Caribe em Ciências da Saúde (LILACS), Biblioteca Virtual de Saúde (BVS) por meio do cruzamento dos seguintes descritores: Cobertura vacinal, Gestantes e Imunização. Foram usados como critérios de inclusão para seleção dos artigos: disponibilidade do texto completo para a leitura, idioma português ou inglês e pertinência ao tema. Como critérios de exclusão foram considerados: artigos pagos e literatura repetida nas bases de dados pesquisadas.

Ademais, foram avaliadas as recomendações nacionais de vacinação, através da busca eletrônica dos Manuais de Vacinação da Federação Brasileira das Associações de Ginecologia e Obstetrícia, da Sociedade Brasileira de Imunização e do Ministério da Saúde.

\section{RESULTADOS E DISCUSSÃO}

A cidade de Ponte Nova/MG, localizada na Zona da Mata Mineira, conta com 4 postos de vacinação, conforme informações extraídas do Sistema do Cadastro Nacional de Estabelecimentos de Saúde (SCNES) (2021). Essas salas de imunização integram as Unidades Básicas de Saúde (UBS) do município e atend em à demanda da população local estimada em 60.003 habitantes (IBGE, 2021). Na Tabela 1 estão descritos os dados coletados referentes à cobertura vacinal das gestantes do município de Ponte Nova/MG, referente ao período de 2018 a 2020, conforme as informações disponibilizadas pelo DATASUS.

Tabela 1 - Coberturas vacinais das gestantes, em Ponte Nova - MG, 2018 a 2020.

\begin{tabular}{|c|c|c|c|}
\hline \multirow{2}{*}{ Vacina } & \multicolumn{3}{|c|}{ Cobertura vacinal (\%) por ano } \\
\hline & 2018 & 2019 & 2020 \\
\hline Influenza gestante & 91,20 & 68,73 & 59,74 \\
\hline Hepatite B & 91,85 & 67,21 & 87,72 \\
\hline Dupla adulto e tríplice acelular gestante & 64,60 & 55,92 & 36,27 \\
\hline dTpa gestante & 71,53 & 77,17 & 58,53 \\
\hline
\end{tabular}

Fonte: Andrade LS e Garcia RMA, 2021; dados extraídos do Departamento de Informática do SUS (DATASUS) e Sistema de Informações do Programa Nacional de Imunizações (SI-PNI).

A partir do ano de 2017, o Ministério da Saúde (2017) estabeleceu como meta vacinar contra o vírus da Influenza pelo menos $90 \%$ dos grupos prioritários, no qual se inclui as gestantes. Os dados dispostos na Tabela 1 demonstram que no município de Ponte Nova/ MG, a meta de vacinação das gestantes contra a Influenza foi atingida apenas no ano de 2018 (91,20\%), ficando abaixo do esperado tanto em 2019 (68,73\%) quanto em 2020, nesse ano, apenas 59,74 das gestantes foram imunizadas contra o vírus da influenza no município.

Quanto à imunização da hepatite $B$, a cobertura vacinal preconizada para a população-alvo (criança, adolescente, adulto, idoso e gestante) é de 95\% (MINISTÉRIO DA SAÚDE, 2015). Apesar do número almejado pelas entidades de saúde, em nenhum dos três anos avaliados o índice de vacinação para a hepatite B foi alcançado no município, cumprindo 91,85\% da cobertura vacinal em 2018. Enquanto no ano de 2019 o índice de cobertura foi $67,21 \%$ e em 2020 de $87,72 \%$. 
A vacina antitetânica (dTpa), por sua vez, é indicada para todas as mulheres em idade fértil (10 a 49 anos de idade), gestantes e não gestantes, e atua como instrumento fundamental para prevenção do tétano neonatal e da coqueluche nos primeiros meses de vida do bebê (MINISTÉRIO DA SAÚDE, 2014). O esquema vacinal a ser realizado, dependerá diretamente da história de imunização antitetânica prévia da gestante comprovada através do cartão vacinal. No munícipio de Ponte Nova/ MG, a cobertura vacinal das gestantes que receberam a vacina dupla bacteriana (dT) e a Tríplice bacteriana acelular durante os anos de 2018, 2019 e 2020 foi de $64,60 \%, 55,92 \%$ e $36,27 \%$, respectivamente.

Já o número de gestantes em Ponte Nova/ MG, que receberam apenas uma dose de reforço da tríplice bacteriana após $20^{\circ}$ semana de gestação foi de $71,53 \%$ em 2018, 77,17\% em 2019 e de $58,53 \%$ em 2020 . Os índices de vacinação coletados indicaram que nos três anos a cobertura vacinal da dT e da dTpa não cumpriram a meta pactuada que seria imunizar 100\% dessa população (MINISTÉRIO DA SAÚDE, 2015).

Em relação aos índices nacionais, a cobertura vacinal das gestantes no Brasil também tem se apresentando aquém das expectativas, fato que pôde ser constatado através dos dados extraídos do site do DATASUS - TABNET. Tais dados mostram a cobertura vacinal para influenza entre as gestantes no país foi de $80,78 \%$ no ano de 2018, 84,60\% em 2019 e 76,89\% em 2020 . Esses dados indicaram que a adesão vacinal não alcançou o índice desejado de $90 \%$ em nenhum dos anos considerados (MINISTÉRIO DA SAÚDE, 2017).

Quanto à vacina Hepatite $B$, os dados extraídos do DATASUS informam que, no país, durante o ano de 2018 a cobertura da vacinação da população-alvo contra a infecção da hepatite B, incluindo o grupo das gestantes, no país foi de 88,53\%, com queda em 2019 apresentando $70,77 \%$ e $77,04 \%$ no ano de 2020 ficando abaixo dos 95\% de cobertura esperados para essa vacina (MINISTÉRIO DA SAÚDE, 2015).

Por fim, o número de gestantes que receberam as vacinas dT e dTpa entre os anos de 2018 e 2020, no Brasil, é ainda menor. Os índices de cobertura vacinal da dTpa e dT alcançaram 44,99\% no ano de 2018 , $45,02 \%$ em 2019 e em 2020, 22,42\%. Já a porcentagem de gestantes que receberam apenas uma dose da dTpa a partir da $20^{\circ}$ semana de gestação foi de $60,23 \%$ em 2018, 63,23\% em 2019 e de apenas $45,46 \%$ em 2020, demonstrando uma queda expressiva nesse último ano. Dessa forma, em nenhum dos anos investigados as coberturas vacinais destes imunobiológicos conseguiram alcançar a meta pretendida de 100\% (MINISTÉRIO DA SAÚDE, 2015).

A monitorização da CV é uma ferramenta comumente usada pela gestão do Programa Nacional de Imunizações e também pelas Secretarias Estaduais (SES) e Municipais de Saúde (SMS). A CV é definida como percentual de indivíduos da população alvo vacinados avaliando isoladamente cada vacina disponível, através dos valores de cobertura vacinal coletados permite-se identificar a ef etividade do PNI (TEIXEIRA AMS e ROCHA CMV, 2010).

Alcançar altas taxas de CV sempre foi uma característica exemplar do PNI, no entanto, nos últimos anos houve uma queda nacional do número de pessoas adequadamente vacinadas, fato que merece atenção especial dos especialistas e das entidades de saúde (CRUZ A, 2017). Através dos dados divulgados neste trabalho foi possível identificar uma redução de $34,49 \%$ da cobertura vacinal da Influenza em gestantes de Ponte Nova/MG quando comparados os anos de 2018 e 2020, sendo que 2020 foi o que apresentou a menor cobertura para essa vacina entre os três anos avaliados.

Em relação à cobertura vacinal contra a Hepatite $B$, o município de Ponte Nova/MG apresentou uma diminuição de $26,82 \%$ do ano de 2018 para o ano de 2019 e um aumento de $30,51 \%$ na cobertura vacinal do ano de 2019 para o ano de 2020. Apesar dessa elevação, a cobertura vacinal da hepatite B entre a população alvo ainda se manteve inferior ao esperado.

Da mesma maneira, a CV dos imunológicos dupla adulto e tríplice acelular gestante também se mostrou abaixo do esperado, tendo uma queda de $13,43 \%$ entre os anos de 2018 e 2019 e de $35,13 \%$ quando avaliados os anos de 2019 e 2020. Já para a vacina dTpa gestante que é aplicada em dose única para todas as gestantes a partir da $20^{\circ}$ semana de gravidez, as taxas se mostravam abaixo das metas preconizadas 
tanto nos anos de 2018 quanto no ano de 2019, tendo uma queda importante no ano de 2020. Entre os anos de 2019 e 2020 houve uma queda de $24,15 \%$ da cobertura vacinal da vacina dTpa gestante.

Conclui-se assim, que coberturas das vacinas preconizadas para as gestantes no município ficaram abaixo dos valores esperados no município de Ponte Nova - MG, em todos os anos avaliados, com única exceção para a vacina da Influenza com alcance da meta esperada (igual ou acima dos $90 \%$ ) no ano de 2018 (MINISTÉRIO DA SAÚDE, 2017).

A queda importante dos índices de cobertura vacinal entre o grupo das gestantes expõe uma realidade preocupante para a saúde coletiva, uma vez que o não alcance de coberturas adequadas de vacinação entre as gestantes está diretamente associada à dificuldade em eliminar o tétano materno e neonatal e, consequentemente, no reaparecimento e vulnerabilidade da população às doenças já controladas por meio da vacinação (MASUNO K, et al., 2009).

Assim como o tétano, a hepatite B é outra doença que pode acometer a gestante e ao seu filho, isso porque mulheres férteis quando contaminadas podem transmitir o vírus da hepatite $B$ para os seus bebês. De acordo com as informações fornecidas pela Federação Brasileira das Associações de Ginecologia e Obstetrícia (FEBRASGO) em 2017, na ocorrência da transmissão vertical desse vírus durante o período perinatal quase $25 \%$ das crianças contaminadas desenvolverão a infecção hepática crônica. Ademais, os bebês poderem evoluir com complicações e vir a óbito em decorrência do carcinoma hepatocelular ou de uma cirrose.

Além disso, as alterações fisiológicas do ciclo gravídico-puerperal tornam as gestantes e seus fetos o grupo populacional com maior risco de complicações na ocorrência de doenças imunopreveníveis e potencialmente fatais. Estudos previamente publicados demonstraram que as gestantes possuem suscetibilidade aumentada de desenvolver doença grave e complicações relacionadas à infecção pelo vírus influenza como hospitalização, admissão em Unidades de Terapia Intensiva e óbito principalmente no terceiro trimestre e no primeiro mês após o parto (TAN TQ, et al., 2017).

Não obstante, a infecção pelo vírus da influenza também pode trazer problemas ao recém-nascido, de acordo com o que foi descrito no estudo de Kachikis A, et al. (2018) podem ocorrer, por exemplo, aumento da ocorrência de baixo peso ao nascer, o óbito fetal e o acréscimo em $30 \%$ no risco de prematuridade (FEBRASGO, 2017). Visto isto, a vacinação da gestante também traz benefícios ao RN e/ou lactente conferindo proteção a esses pelo menos até os seis meses de vida enquanto esses indivíduos ainda não foram imunizados (MERTZ D, et al., 2017).

Embora existam inúmeras campanhas e ações que incentivem a vacinação durante a gravidez, vários obstáculos continuam influenciando na adesão das gestantes a imunização. As falhas de comunicação, a veiculação de "fake news", além da falta de conhecimento a respeito dos benefícios da vacinação e dos possíveis riscos na ocorrência de um processo infeccioso são condições que induzem muitas pessoas a rejeitarem a vacinação, fato que culmina em queda da cobertura vacinal e em declínio da imunidade coletiva (COLGROVE J, 2016).

Uma recente revisão literária desenvolvida por Lutz CS, et al. (2018) apontou os inúmeros fatores que podem afetar a vacinação na gravidez, através desta foram identificadas as barreiras referentes ao paciente e as barreiras relacionas ao provedor de saúde. Dentre os obstáculos relacionados às gestantes, as preocupações com a segurança da mãe e/ou do recém-nascido foram às queixas mais citadas. Outras questões frequentemente comentadas incluem as preocupações maternas sobre a eficácia das vacinas, a falta de percepção das gestantes com relação gravidade das doenças, além da falsa ideia de que pessoas saudáveis não precisavam receber a vacina também estão associados a essa falta de adesão

Quanto aos provedores de saúde, além do pouco conhecimento sobre as recomendações para a imunização das gestantes, outras barreiras comumente citadas estão relacionas às preocupações financeiras, além da incapacidade de solicitar, obter e armazenar as vacinas (LUTZ CS, et al., 2018). Em concordância, um estudo atualizado sobre o assunto indica que a falta de conhecimento dos profissionais de saúde e das 
gestantes sobre vacinas preconizadas é um dos principais entraves para o alcance de coberturas adequadas de imunização (ABU-RAYA B, et al., 2020). Por esse motivo, a equipe que atua nos cuidados à saúde da mulher durante a preconcepção ou pós-concepção deve estar em integração para não perderem a oportunidade de orientar e vacinar as gestantes no momento correto (MINISTÉRIO DA SAÚDE, 2013).

Além dos inúmeros obstáculos pré-existentes para a vacinação das gestantes somou-se ainda o atual cenário pandêmico da COVID-19. A doença que já matou milhões de pessoas pelo mundo e mudou todo o cenário global de saúde exerceu influência direta nos índices de imunização das mulheres grávidas, como prova disso, durante o ano de 2020 menos de $50 \%$ das mulheres grávidas brasileiras receberam as vacinas preconizadas durante esse período (FEBRASGO, 2020).

No intuito de vencer os obstáculos que limitam o alcance de coberturas vacinais preconizadas para as gestantes e melhorar a aceitação materna a imunização, o American College of Obstetricians and Gynecologists (ACOG) (2010), através do Comitê de Integração de Imunização sugere algumas medidas fundamentais para esse processo, sendo elas: a educação, a recomendação, a normatização e a melhora da conveniência.

De acordo com o ACOG (2010), a abordagem e o esclarecimento sobre os risco e benefícios da vacinação constituem passos importantes para conquistar a confiança das gestantes e, consequentemente, melhorar a adesão e a procura ativa pela vacinação. A recomend ação diz respeito à relação médico/ paciente visto que a comunicação verbal de um médico parece ser o maior motivador para aceitação das vacinas das gestantes.

Ainda conforme o ACOG (2010), a normatização incentiva médicos ginecologista-obstetras e generalistas a adotarem uma rotina ou protocolo, ainda em sua primeira consulta de preconcepção e, principalmente, de pré-natal, visando à abordagem da prevenção de doenças inf ecciosas maternas e infantis, além de avaliar os cartões de vacina de cada gestante e, caso necessário, indicar as vacinas que estão em falta. E em relação á conveniência, se a vacinação for realizada diretamente no local onde é realizada a consulta de pré-natal, torna-se mais fácil imunizar a gestante logo no momento pós consulta, quando ela passou pelo médico, recebeu as informações necessárias e, a partir daí sendo encaminhada para as salas de vacina.

Diante de todas as questões apresentadas, a consolidação e análise dos dados apresentados, referentes à imunização das gestantes no município de Ponte Nova/ MG e também no território nacional fornecerão meios para identificação do problema e, consequentemente, contribuem para a definição de estratégias de atuação na tentativa de solucionar as barreiras limitantes à imunização das gestantes de modo a incentivar a adesão materna e o aumento de sua cobertura vacinal.

\section{CONCLUSÃO}

Este trabalho promoveu a exposição e análise de dados atuais referentes à cobertura vacinal das gestantes no município de Ponte Nova/MG e no território nacional, em que os resultados encontrados mostram baixa adesão das gestantes à imunização e apontam para a necessidade de maior divulgação de informações e publicações científicas sobre o assunto, no intuito de esclarecer possíveis dúvidas, desmistificar Fake News, e ainda demonstrar os benefícios da vacinação para este público. Diante disso, enfatiza-se também a importância da manutenção e ampliação de políticas públicas cuidadosamente destinadas à melhoria do atendimento materno, com investimentos em ações voltadas para educação em saúde das gestantes e capacitação dos profissionais da saúde.

\section{REFERÊNCIAS}

1. ABU-RAYA B, et al. Global perspectives on immunization during pregnancy and priorities for future research and development: an international consensus statement. Front Immunol, 2020;11:1282.

2. AMERICAN COLLEGE OF OBSTETRICIANS AND GYNECOLOGISTS (ACOG). Committee Opinion No. 468: Influenza vaccination during pregnancy. American College of Obstetricians and Gynecologists Com mittee on Obstetric Practice. Obstet. Gynecol., 2010; 116(4):1006-1007. 
3. COLGROVE J. Vaccine Refusal Revisited - The limits of public health persuasion and coercion. N Engl. J. Med, 2016; 375(14): 1316-1317.

4. CRUZ A. A queda da imunização no Brasil. Redução da cobertura vacinal no país é preocupante. Revista Consensus, 2017. Disponível em: https://www.conass.org.br/consensus/queda-da-imunizacao-brasil/. Acessado em: 29 ago. 2021.

5. FEDERAÇÃO BRASILEIRA DAS ASSOCIAÇÕES DE GINECOLOGIA E OBSTETRÍCIA (FEBRASGO). A vacinação da gestante. 2017. Disponível em: https://www.febrasgo.org.br/pt/noticias/item/199-a-vacinacao-dagestante?highlight=WyJ2YWNpbmFcdTAwZTdcdTAwZTNvliwiZGFzliwiZ2VzdGFudGVzliwidmFjaW5hXHUwMGU3 XHUwMGUzbyBkYXMiLCJ2YWNpbmFcdTAwZTdcdTAwZTNvIGRhcyBnZXNOYW50ZXMiLCJkYXMgZ2VzdGFudG $\mathrm{VzllO}=$. Acessado em: 15 ago. 2021.

6. FEDERAÇÃO BRASILEIRA DAS ASSOCIAÇÕES DE GINECOLOGIA E OBSTETRÍCIA (FEBRASGO). Covid: Febrasgo comenta se a vacina pode ser utilizada por gestantes. 2020. Disponível em: https://www.febrasgo.org.br/pt/noticias/item/1198-covid-febrasgo-comenta-se-a-vacina-pode-ser-utilizada-porgestantes. Acessado em:14 ago. 2021.

7. INSTITUTO BRASILEIRO DE GEOGRAFIA E ESTATíSTICA (IBGE). Censo demográfico. Disponível em: https://cidades.ibge.gov.br/brasil/mg/ponte-nova/panorama. Acessado em:27 set. 2021.

8. KACHIKIS A, et al. Who's the Target: Mother or Baby? Viral Immunol., 2018;31(2):184-194.

9. LAJOS GJ, et al. Imunização na gravidez, puerpério e amamentação. In: Programa vacinal para mulheres. São Paulo: Federação Brasileira das Associações de Ginecologia e Obstetrícia (FEBRASGO). Série Orientações e Recomendações FEBRASGO; $n^{\circ} .13$, Comissão Nacional Especializada de Vacinas. 2017; 14: 128-138. Disponível em:https://sogirgs.org.br/area-do-associado/programa-vacinal-para-mulheres.pdf. Acesso em:1 abr. 2021.

10. LOUZEIRO EM, et al. A importância da vacinação em gestantes: uma revisão sistemática da literatura no período de 2003 a 2012. Rev Interd, 2014; 7(1):193-203.

11. LUTZ CS, et al. Understanding barriers and predictors of maternal immunization: identifying gaps through an exploratory literature review. Vaccine, 2018, 36: 7445-7455.

12. MASUNO K, et al. Scaling up interventions to eliminate neonatal tetanus: factors associated with the coverage of tetanus toxoid and clean deliveries among women in Vientiane, Lao PDR. Vaccine, 2009;27(32): $4284-4288$.

13. MERTZ D, et al. Pregnancy as a risk factor for severe outcomes from influenza virus infection: A systematic review and metaanalysis of observational studies. Vaccine, 2017;35(4);521-528.

14. MINISTÉRIO DA SAÚDE. Informe Técnico: 19a Campanha Nacional de Vacinação contra a Influenza. 2017. Disponível em:https://cvpvacinas.com.br/pdf/Informe_Cp_Influenza-2017.pdf. Acesso em:20 abr. 2021.

15. MINISTÉRIO DA SAÚDE. Programa Nacional de Imunização. Calendário Nacional de Vacinação 2020.2020. Disponível https://www.saude.go.gov.br/files/imunizacao/calendario/Calendario.Nacional.Vacinacao.2020.atualizado.pdf. Acesso em: 1 mar. 2021.

16. MINISTÉRIO DA SAÚDE. Programa Nacional de Imunizações 30 anos. 2003. Disponível em: https://bvsms.saude.gov.br/bvs/publicacoes/livro_30_anos_pni.pdf. Acesso em:14 mar. 2021.

17. MINISTÉRIO DA SAÚDE. Secretaria de Atenção à Saúde. Departamento de Atenção Básica. Atenção ao pré -natal de baixo risco. 2013. Disponível em:http://bvsms.saude.gov.br/bvs/publicacoes/atencao_pre_natal_baixo_risco.pdf. Acessado em:14 mar. 2021.

18. MINISTÉRIO DA SAÚDE. Secretaria de Vigilância em Saúde. Departamento de Vigilância das Doenças Transmissíveis. Manual de Normas e Procedimentos para Vacinação. 2014. Disponível em: https://portaldeboaspraticas.iff.fiocruz.br/wp-content/uploads/2021/04/manual_procedimentos_vacinacao.pdf. Acesso em:20 abr. 2021.

19. MINISTÉRIO DA SAÚDE. Secretaria de Vigilância em Saúde. Programa Nacional de Imunizações. Coberturas vacinais no Brasil. Período $2010 \quad-\quad 2014.2015 .2$ Disponível em: https://portalarquivos2.saude.gov.br/images/pdf/2017/agosto/17/AACOBERTURAS-VACINAIS-NO-BRASIL---20102014.pdf. Acessado em 20 abr. 2021.

20. SISTEMA DE INFORMAÇÕES DO PROGRAMA NACIONAL DE IMUNIZAÇÕES (SI-PNI). 2020. Disponível em: http://sipni-gestao.datasus.gov.br/si-pni-web/faces/relatorio/consolidado/vacinometrolnfluenza.jsf. Acesso em: 15 maio 2021.

21. SISTEMA DO CADASTRO NACIONAL DE ESTABELECIMENTOS DE SAÚDE (SCNES). 2021. Disponível em http://cnes.datasus.gov.br/pages/estabelecimentos/consulta.jsp. Acessado em:29 jul. 2021.

22. TAN TQ, et al. The Vaccine Handbook: A Practitioner's Guide to Maximizing Use and Efficacy across Lifespan.1st ed. New York: Oxford University Press, 2017.

23. TEIXEIRA AMS, ROCHA CMV. Vigilância das coberturas de vacinação: uma metodologia para detecção e intervenção em situações de risco. Epidemiol. Serv. Saúde, 2010; 19(3):217-226. 